\title{
Evaluating the Dimensionality of Pornography
}

Dean M. Busby

Brigham Young University - Provo, dean_busby@byu.edu

Hsin-Yao Chiu

Brigham Young University - Provo

Joseph A. Olsen

Brigham Young University - Provo

Brian J. Willoughby

Brigham Young University - Provo

Follow this and additional works at: https://scholarsarchive.byu.edu/facpub

Part of the Other Social and Behavioral Sciences Commons

\section{Original Publication Citation}

Busby, D. M., \& Chiu, H-C., Willoughby, B. J., Olsen, J. A. (2017). Evaluating the dimensionality of pornography. Archives of Sexual Behavior, 46, 1723-1731.

\section{BYU ScholarsArchive Citation}

Busby, Dean M.; Chiu, Hsin-Yao; Olsen, Joseph A.; and Willoughby, Brian J., "Evaluating the Dimensionality of Pornography" (2017). Faculty Publications. 4628.

https://scholarsarchive.byu.edu/facpub/4628

This Peer-Reviewed Article is brought to you for free and open access by BYU ScholarsArchive. It has been accepted for inclusion in Faculty Publications by an authorized administrator of BYU ScholarsArchive. For more information, please contact ellen_amatangelo@byu.edu. 


\title{
Evaluating the Dimensionality of Pornography
}

\author{
Dean M. Busby ${ }^{1} \cdot$ Hsin-Yao Chiu $^{1} \cdot$ Joseph A. Olsen $^{1} \cdot$ Brian J. Willoughby $^{1}$
}

Received: 2 March 2016/Revised: 17 March 2017 / Accepted: 24 March 2017 / Published online: 31 March 2017

(C) Springer Science+Business Media New York 2017

\begin{abstract}
Pornography may be a construct with a single trait or one with many traits. Research in the past was inconsistent in this regard with most researchers assuming that pornography was unidimensional (with one single trait of pornography). However, the considerable amounts of residual variation found in these studies beyond that explained by the single trait hints at what might be a multidimensional construct (with multiple traits such as sensitization and differentiation). Consequently, in this study, we intended to address the question of whether pornography consisted of a single trait or if it was multidimensional. Using MTurk, 2173 participants from the United States and the Commonwealth of Nations (in which pornography is not strictly illegal) were recruited and asked to rate how pornographic they thought a list of different depictions were. The data were analyzed utilizing the cross-validation procedure in which two subsamples were created from the main sample and one was used to establish the model building and the other to validate the model. Various models, including first-order and higher-order exploratory and confirmatory factor models, were tested. Results indicated that a bi-factor (multidimensional) model generated the best model fit, and that it was most appropriate to consider pornography multidimensional. The final model contained two dimensions ("Sensitization" and "Differentiation"). While sensitization revealed the participants' general tendency to rate all items to be more or less pornographic, differentiation revealed the participants' tendency to differentiate highly pornographic items from less pornographic items. Based on the findings of this study, we suggest that future research on the usage and effects of pornography be con-
\end{abstract}

Dean M. Busby

dean_busby@byu.edu

1 School of Family Life, Brigham Young University, 2086 JFSB, Provo, UT 84602, USA ducted while taking into consideration the multidimensional nature of pornography.

Keywords Pornography · Erotica - Sexually explicit media . Multidimensionality

\section{Introduction}

With the vast growth of technology, pornography is now more easily accessible than ever and has thus become an expanding area of research in recent years (e.g., Manning, 2006; Wright, 2013). Despite the growing body of literature, a fundamental debate remains: What defines pornography? If we are to define, understand, and measure pornography it is essential to address a relatively unanswered question: Is pornography a unidimensional or multidimensional construct?

Research efforts on pornography started in the 1960s and have accelerated in number to the present (Hald, Seaman, \& Linz, 2014). Research participants represent a wide age range (e.g., Hald \& Mulya, 2013; Tsaliki, 2011) recruited throughout the world (e.g., D'Abreu \& Krahé, 2014; Subero, 2010; Wright, Tokunaga, \& Bae, 2014; Yu, 2013). Various outcome variables have been associated with pornography use from personal well-being to a variety of interpersonal relationship measures (e.g., Poulsen, Busby, \& Galovan, 2013). However, despite the abundant research efforts on pornography, there continue to be challenges, such as the fact that many study results conflict with one another. For example, as Hald et al. (2014) pointed out, while some scholars provided evidence that pornography could be harmful, other researchers claimed the opposite. Differences in research methods could be one source of the inconsistency in the findings, and one of the most widely acknowledged differences is how pornography is measured. 


\section{The Definition of Pornography Based on Wording}

Several scholars have pointed out that the measurement issue is fundamental to pornography research (Hald et al., 2014; Malamuth, Addison, \& Koss, 2000; Short, Black, Smith, Wetterneck, \& Wells, 2012; Willoughby \& Busby, 2016). Specifically, the lack of a common definition for pornography remains unresolved. In many instances, researchers allow participants to define pornography on their own (e.g., Sinković, Štulhofer, \& Božić, 2013; Tylka, 2015; Wright, 2013). This leads to an apparent problem: Participants have widely varying definitions of what constitutes pornography depending on their gender, religiosity, marital status, and other factors (Willoughby \& Busby, 2016). Also, a lack of common definition makes it nearly impossible to compare results across studies (Short et al., 2012).

While self-defined pornography measurements are prevalent, there are also studies that utilize more specific approaches, such as: (1) human and non-human images, including strangers, teachers, and actresses (Yu, 2013); (2) the types of media, including TV, internet, cell phone, books (D'Abreu \& Krahé, 2014); (3) the types of sexual interactions involved, including kissing and vaginal intercourse (Yu, 2013); (4) the ratings of the movie (Wright et al., 2014); and (5) whether the intention of the media is to create or enhance sexual feelings or thoughts (Hald \& Mulya, 2013). Although it is clear from these studies that there are a number of ways researchers have defined pornography, there is little consistency between scholars. Furthermore, many of the measurements are not standardized or validated, which again may lead to a lack of consistency across research findings (Short et al., 2012).

\section{The Definition of Pornography Based on Dimensionality}

Beyond the issues addressed above, we noticed yet another theme that researchers have begun to discuss when defining pornography: What is the dimensionality of the construct? Dimensionality refers to the number of basic underlying subcomponents that are needed to empirically assess the primary construct domain. At times, dimensionality can be found through (but does not always correspond precisely with) the number of factors obtained from a factor analysis of the items. As established in the literature, some have presumed or concluded that appraisals of different sexual enactments follow a common unidimensional, single factor continuum. For example, in some of the early studies from the 1970s, researchers treated pornography more as a single factor (unidimensional) construct, going from soft to hard core (e.g., Amoroso, Brown, Pruesse, Ware, \& Pilkey, 1972; Turnbull \& Brown, 1977). Meanwhile, others emphasized a multifactorial (but not necessarily multidimensional) account of pornography judgments.
For example, Brown, Anderson, Burggraf, and Thompson (1978) found four distinct factors ordered along one single dimension from 25 pornographic images. Yet, these 25 pictures were found to be unidimensionally scalable. Likewise, Dalecki and Price (1994) and Price and Dalecki (1998) identified five factors from 22 sexual images. These foundational studies, although important, are not commonly referenced in the more recent pornography literature. Most current studies consider pornography unidimensional and with only one factor.

As for the statistical methods that have been used in the past to study the dimensionality of pornography, previous multifactorial studies (e.g., Brown et al., 1978; Dalecki \& Price, 1994) have required the multiple factors to be mutually orthogonal through the use of varimax rotation following principal component analysis or exploratory factor analysis. Varimax rotation attempts to achieve simple structure of the factor loadings where each item has a substantial loading on just one factor, while simultaneously requiring the factors themselves to be uncorrelated with each other. This means that although pornography was considered to be a multifactorial construct, a multidimensional alternative was not considered. For example, 16 of the 25 items in Brown et al. (1978) and 9 of the 22 items in Dalecki and Price (1994) had secondary loadings above. 30 on one or more of the other orthogonal factors. The presence of numerous large secondary factor loadings in the prior studies using varimax rotation provides an initial indication that a simple structure model with uncorrelated factors may not represent the data well and that pornography may be multidimensional in nature.

In summary, after considering what has been done and what was found regarding the definition of pornography, it is clear that we need to further understand and explore potential items and measures through more complex means than has been previously done.

\section{Purpose of Study and Research Questions}

In this study, we sought to extend the literature by defining pornography through examining the perceptions of what participants considered to be pornography, as well as identifying the dimensionality of pornography through various statistical models. We accomplished our purposes by asking participants to rate how pornographic a variety of scenarios were. Then, we explored the dimensionality of pornography by using exploratory and confirmatory factor analyses to analyze the data, relaxing the assumptions of simple structure used in the previous research. While based on sparse previous research, we expected pornography to be multidimensional. The question of dimensionality was crucial because if pornography was multidimensional, the instruments used to evaluate it must measure those dimensions effectively before the effects and predictors of pornography can be understood. 


\section{Method}

\section{Sample and Procedure}

The sample was collected through Amazon Mechanical Turk (www.mturk.com), also known as MTurk. MTurk is a Web site that provides a worldwide online platform for the completion of Human Intelligence Tasks (HITs), such as opinion surveys. On this Web site, the HIT "requesters" provide projects, and the qualified HIT "workers" select and finish the job tasks to receive payment. For the purpose of our study, we required that the participants were English speaking and at least 18 years old. The HIT task that we posted was a 10-15min survey asking about demographic information, usage of pornography, and attitudes regarding the definition of pornography. Prior to the survey, the participants were asked to read and agree to a consent form. Those that agreed to the consent request then moved on to answering the survey. Upon completion of the survey, each participant was compensated with $\$ 0.25$, which was similar to comparable task payments on MTurk.

The sample used in this study consisted of 2173 individuals. Originally, 2459 participants from more than 45 different countries took the survey. Because we were concerned that the diverse countries may introduce potential problems of the participants not having sufficient English proficiency, we only retained participants from the United States and the Commonwealth of Nations where English was either the primary language used or was at least one of the official languages of the country. Furthermore, we eliminated participants from the countries where pornography was illegal. Since our measures assessed participants' perceptions of pornography, in countries where pornography was considered illegal the participants may have different moral or legal standards regarding pornography than the rest of the participants and thus skew the results. Finally, we removed 217 participants who did not finish the survey and missed over $20 \%$ of the questions.

Participants ranged from 18 to 73 years old, with a mean age of 30.7. Among all participants, $64.1 \%$ were males, $35.3 \%$ were females, and .6\% were transgender. Regarding their occupational and/or educational status, 5\% were full-time students and not employed, $53 \%$ were working full-time and not going to school, and the rest were a mixture of part-time workers and/ or part-time students. As far as their highest level of education, $23 \%$ had some college or less, $52 \%$ had 4 - or 2-year degrees, and $25 \%$ had masters or doctoral degrees. Fifty-three percent of the participants were living in the United States, $23 \%$ were in India, $2 \%$ in Canada, and the remaining $22 \%$ were in other countries of the Commonwealth of Nations, such as Australia, New Zealand, Sweden, and the United Kingdom. Finally, in terms of the pornography use of these participants, when asked how often in the last 12 months did they "view a video showing a graphic sexual act or fully nude people," $21 \%$ answered by choosing the category "none," $28 \%$ chose "once a month or less," $19 \%$ chose " 2 or 3 days a month," $16 \%$ chose " 1 or 2 days a week," $10 \%$ chose " $3-5$ days a week," and $6 \%$ chose "almost everyday".

In this study, we utilized the cross-validation strategy to check for potential over-fitting problems. To do so, we randomly split the total sample into two halves. One half of the sample (Sample A, $n=1105$ ) was used to conduct preliminary analyses and to build exploratory/confirmatory factor models. We then used the second half of the sample (Sample B, $n=1068$ ) to replicate and validate the confirmatory factor analysis.

\section{Measures}

\section{Perceptions of Pornography}

In this study, we used a set of items that were developed by our research team (Willoughby \& Busby, 2016) to measure the survey respondents' perceptions and definitions of pornography. We used perceptions instead of behaviors because individuals are likely to have perceptions about what pornography is regardless of whether they might have used different types of sexual media. After reviewing a variety of previous studies on pornography and pornography measurements, our team created 20 items that captured a wide range of media depictions that could be considered pornography. Although more than 20 items could have been added which might have covered a plethora of pornographic materials and more mediums of delivery, we selected only 20 items to reduce potential participant fatigue and irritation with perceived redundancy.

These 20 items described pornographic situations by media types and the intensity of sexuality portrayed. We asked the participants to "consider the statements below and indicate if you believe they describe what you would consider pornography" on an 11-point Visual Analog Scale (see Dimitrov, 2012) from 0 to 10 , with 0 representing "definitely not pornography" and 10 representing "definitely pornography." This scale appeared in the format of a sliding bar. As participants moved the sliding bar toward either direction ( 0 or 10$)$, a number also appeared in a dialogue box on the side to inform the participants of the exact number on the scale that the sliding bar was landing on. For a detailed list of all 20 items, please refer to the "Appendix." For this current study, we only used 19 items and left out item 3 ("A video showing a woman having sexual intercourse with an animal") because of low variability and poor content validity as it was the only item that included something other than humans. Although the entire survey that participants completed included more than the "Perceptions of Pornography" items and missing data existed for many of the questions, for this specific measure there were no missing data.

In a previous study using the same survey (Willoughby \& Busby, 2016), we showed some relationships between demographic predictors (including gender, religiosity, marital status, 
and pornography use) and the participants' perceptions regarding pornography. For detailed analyses and results, please refer to Willoughby and Busby.

\section{Analysis}

All of the analyses for this study were conducted in MPlus version 7.3.

\section{Exploratory Factor Analysis}

Using the model-building sample (Sample A), we conducted a series of exploratory factor analyses (EFA) to examine the overall performance of the items of the pornography measure. Through these exploratory factor analyses, we examined several possibilities to model our data, including a multifactorial model with first-order factor analyses (which focused on loading the 19 items on a number of first-order factors), and multidimensional or higher-order factor analyses, including a bi-factor analyses (in which some of the items loaded on more than one factor).

For the purposes of this study, we distinguish between the term "multifactorial" and "multidimensional." In the current article, we use the term "multifactorial" to refer to a construct that has several factors but items only load on one factor or subscale. In contrast, we use the term "multidimensional" to mean that a construct has more than one dimension because the same items can load on more than one factor. While the terminologies used in the literature may not always be consistent, the concept of multifactors versus multidimensions is not new. For example, Hartig and Höhler (2008) discussed the construct of multifactorial and multidimensional models by addressing them as within-item and between-item multidimensional models. A between-item multidimensional model has all items measuring different sets of latent traits. A within-item model, on the other hand, requires items to load on more than one dimension. Hartig and Höhler further clarified that the difference between the within-item model and between-item model is whether the items load on more than one trait. While the terminologies may be diverse, the central idea is consistent: That there is a difference between multifactorial constructs and multidimensional constructs.

As mentioned previously, as part of EFA, we tested the possibility of modeling our data using a bi-factor model. A bifactor model is a factor structure that is less common than typical factor structures. In a bi-factor model, items can load on multiple factors simultaneously, and is thus a type of multidimensional construct. Bi-factor analyses are appropriate when researchers hypothesize that there is one general factor accounting for the commonalities among all items, and meanwhile additional domain specific factors are hypothesized to account for the additional influences of the items (Chen, West, \& Sousa, 2006). As we continue to discuss throughout this study, our pornography items reflected various levels of complexity. Since all of our items were designed to capture a specified latent concept (namely pornography), we expected to find a primary common latent variable that was reflected by all of the items. However, we were also exploring any unexplained covariation among the items not captured by the main common factor. We wondered about possible additional underlying latent factors for these items because the items not only described the central concept of pornography, but also contained other themes such as types of media and intensity of sexualized messages. Because we expected the items to reflect a common underlying construct, along with possible effects of additional latent factors, the use of bi-factor multidimensional analysis was appropriate.

\section{Confirmatory Factor Analyses}

In order to confirm that this bi-factor model was an appropriate model for our data, we conducted a confirmatory factor analysis also using Sample A. In a bi-factor model where all items load on both factors, to identify the model it is required that at least one item loading on the second factor be constrained to zero (Bollen, 1989). According to the ESEM model outcome, the loading of Item 8 on the second factor had the loading closest to zero. As a result, we chose to constrain the factor loading of Item 8 on the second factor as zero in the confirmatory factor analysis.

The confirmatory factor analysis results indicated that the model fit did not change after fixing the loading of Item 8 on the second factor to zero. The confirmatory factor analysis also indicated good fit. Since the model fit indices were essentially identical for both models, we decided to retain the ESEM model as our final model, which provided us with estimates of factor loadings of all items on both factors. As a final step, we replicated the results using the validating sample (Sample B).

\section{Results}

Our primary research interest was whether pornography (from the perceptions of the study participants) was unidimensional or multidimensional. In order to examine this, we conducted factor analyses in which we utilized exploratory and confirmatory analyses to test several models.

\section{Exploratory Factor Analyses Results}

For the exploratory factor analyses results, we modeled one to six factors for the 19 items in MPlus using Sample A (see Table 1). Various criteria for selecting the appropriate number of factors and for judging model fit provided different suggestions concerning these data. The traditional proportion of variance of .50 and the SRMR of 05 for well-fitting models suggested the necessity of two factors. The traditional scree, eigenvalue of one, and CFI and TLI of .90 for adequately fitting models suggested the need for three factors. The CFI of .95 for well-fitting models and 
Table 1 Exploratory factor analysis and the number of factors using sample A

\begin{tabular}{llllllll}
\hline Number of factors & Eigenvalue & Proportion of variance & CFI & TLI & RMSEA & SRMR & Number of parameters \\
\hline 1 & 7.80 & .41 & .58 & .52 & .18 & .16 & 57 \\
2 & 3.61 & .60 & .89 & .86 & .10 & .04 & 75 \\
3 & 1.08 & .66 & .93 & .90 & .09 & .03 & 92 \\
4 & .80 & .70 & .96 & .93 & .07 & .02 & 108 \\
5 & .73 & .74 & .98 & .96 & .06 & .02 & 123 \\
6 & .58 & .77 & .99 & .97 & .05 & .01 & 137 \\
\hline
\end{tabular}

$N=1105$

the RMSEA of .08 for adequately fitting models suggested four factors. The TLI of .95 for well-fitting models suggested five factors, and the RMSEA of .05 for well-fitting models suggested six factors. Regardless of the various interpretations provided by model fit information, one implication in common was that one factor was too few and at least two factors were required, indicating the possible multidimensional nature of the construct. The need for more than one factor indicates that pornography was multitrait and possibly multidimensional.

While minimally two factors were required, six factors were likely too many. However, in attempting to further narrow the number of latent variables, standard exploratory factor analysis is unlikely to provide an adequate guide to the dimensionality of the data when models with certain rotations, different restrictions on factor loadings, and the possibility of correlated errors were considered. Therefore, after having posited the importance of a common general factor affecting all of the items, we focused our examination on exploratory multidimensional bi-factor models and rotations, rather than traditional independent cluster or simple unidimensional structure models.

\section{Multidimensional Model Results}

The first multidimensional model we evaluated (using Sample A) had four orthogonal factors using a BI-GEOMIN rotation that exhibited adequate model fit and provided a theoretically interpretable loading pattern (see Table 2). Model fit information showed that $\chi^{2}=13458.50, p<.001, \mathrm{CFI}=.96, \mathrm{TLI}=$ $.93, \mathrm{RMSEA}=.07, \mathrm{SRMR}=.02$. In this model, each of the items was permitted to load on all four factors. All of the items had substantial loadings on the first factor. We labeled the first (general) factor Sensitization. We believed it represented the participants' tendency to give higher versus lower ratings to all of the items, reflecting their generalized responsiveness to the portrayal of sex and sexuality in media. This idea of general sensitization is most clearly illustrated by the fact that the factor loadings for the sample are relatively uniform and not dramatically different based on characteristics of the items.

We labeled the second (bipolar) factor Differentiation. We believed it reflected the participants' propensity to discriminate between differing levels of sexual explicitness. This means that this factor reflected the participants' tendency to rate individual items as more or less pornographic based on their ability to differentiate between various items. The differentiation factor thus showed different levels of sexual explicitness described by groups of items. For example, Items 6, 12, 19, and 20 had the strongest positive loadings, which indicated that these items were more prone to be perceived as highly pornographic media. On the other hand, Items 2, 4, 5, and 11 had substantial negative loadings, indicating that participants considered these items to be less pornographic when compared with other items in the scale. The rest of the 11 items were spread between the highly sexually explicit and less sexually explicit groups of items. However, they still represented at least two different themes or levels of sexual explicitness. For example, Items 1, 9, 10, 14, 16, and 17 had minor positive loadings, and shared a common theme of nudity. Meanwhile, Items 7, 13,15 , and 18 had minor negative loadings. These items appeared to include some form of writing or indirect depiction of sexuality. Thus, the differentiation factor represents individual differences in the tendency to discriminate between more sexually explicit and less sexually explicit media in making judgments about pornography.

Together, these two factors (sensitization and differentiation) accounted for the majority of the variation and covariation among the items. Exploratory factor analyses results indicated that the proportion of variance for a two-factor model was .60, while the proportion of variance for the four-factor model was .70 .

The third and fourth factors were most plausibly interpreted as method factors, reflecting shared similarity of item wording and affecting these two different sets of items beyond the main substantive sensitization and differentiation constructs. For example, Items 7, 13, and 15 had the highest factor loadings on the third factor $(.39, .35$, and .37 , respectively). When examining these items, they all contained a common theme of pornography in written descriptions. Also, Items 8, 9, and 16 (with factor loadings of $.33, .37$, and .34 , respectively, on the fourth factor) all shared the common theme of a model with suggestive poses in an image. As pointed out by Brown (2015), such phenomena could merely be method effects that reflect the artifact of scale development 
Table 2 Factor loadings of exploratory four-factor model with bi-factor rotation using sample A

\begin{tabular}{|c|c|c|c|c|}
\hline Item & Factor 1 (sensitization) & Factor 2 (differentiation) & Factor 3 (methods) & Factor 4 (methods) \\
\hline 2 & .66 & -.53 & -.03 & -.18 \\
\hline 4 & .64 & -.56 & .00 & -.24 \\
\hline 5 & .71 & -.46 & -.04 & -.09 \\
\hline 11 & .70 & -.35 & .09 & -.01 \\
\hline 7 & .57 & -.06 & .39 & -.02 \\
\hline 13 & .63 & -.16 & .35 & -.03 \\
\hline 15 & .64 & -.07 & .37 & .01 \\
\hline 17 & .59 & .18 & .44 & .02 \\
\hline 18 & .70 & -.22 & .26 & .02 \\
\hline 1 & .59 & .04 & -.11 & .14 \\
\hline 8 & .78 & -.04 & -.12 & .33 \\
\hline 9 & .72 & .19 & -.06 & .37 \\
\hline 10 & .63 & .27 & -.01 & .22 \\
\hline 14 & .64 & .24 & .09 & .25 \\
\hline 16 & .68 & .15 & .06 & .34 \\
\hline 6 & .41 & .66 & -.08 & .00 \\
\hline 12 & .31 & .78 & .02 & -.06 \\
\hline 19 & .47 & .63 & .02 & .06 \\
\hline 20 & .37 & .75 & -.05 & -.13 \\
\hline
\end{tabular}

rather than conceptually useful dimensions. As suggested by Brown, while adding such additional factors will always improve the model fit, the question is whether these additional factors are truly tapping into important underling constructs or whether they compromise construct validity.

Since the results we obtained from the first multidimensional model with four factors (using Sample A) showed that the two primary factors accounted for most of the variance in the model and the other two factors could be due to methods effects, in the interests of parsimony we thus tested a model (still using Sample A) with only the first two factors. Model fit information showed that $\chi^{2}=$ $1563.55, p<.001, \mathrm{CFI}=.89, \mathrm{TLI}=.86, \mathrm{RMSEA}=.10, \mathrm{SRMR}=$ .04. Although as expected, this simplified model did not fit as well as the full four-factor model, it provided a more parsimonious explanation for the patterns in the data. Even though adding the two additional factors would push the fit indices beyond the arbitrary levels considered "good fit," in our opinion the two-factor model is the most theoretically sound and parsimonious model and the third and fourth factors likely do not represent important underlying constructs that are interpretable. As pointed out by Hartig and Höhler (2008), at times models could be equally appropriate for the data, but it is the researchers' duty to choose the most appropriate model based on not only the numbers, but even more so the "intended interpretation of the latent dimensions."

As we finalized a statistical model to conceptualize pornography, we re-tested and validated the same model using Sample B. Model fit information showed that $\chi^{2}=1517.50, p<.001$, $\mathrm{CFI}=.90, \mathrm{TLI}=.87, \mathrm{RMSEA}=.10, \mathrm{SRMR}=.04$, which was similar to the results of the same model using Sample A. Our finalized model with Sample B as well as the detailed factor loadings is shown in Fig. 1.

We concluded from our final bi-factor model that at least based on our samples, the best way to conceptualize pornography is to look at it from two different perspectives or dimensions simultaneously. That is, individuals from our sample demonstrated two distinct processes regarding what pornography was to them. Pornography was both (1) how sensitized participants were toward pornography (and thus rated all items higher or lower on the scale), and (2) how likely participants were to differentiate between varying levels of sexual explicitness of the media.

\section{Discussion}

From the statistics used in this study, it appeared that pornography is likely multidimensional. While a few early studies hinted at the possibility of multiple dimensions (Brown et al., 1978, Dalecki \& Price, 1994), because the statistics were relatively primitive and exploratory, these early findings could best be described as suggesting a multifactorial model rather than a multidimensional model. As described earlier, we are defining multidimensional as different than multifactorial. While simple analyses from previous work have explored and laid an important foundation to establish whether there might be different factors embedded in pornography measures (such as soft-core or hard-core pornography, see 
Brown et al., 1978), we explored in this study a more fundamental question around the key dimensions underlying judgments about pornography. Our initial analyses identified four dimensions as the best fitting model, but the most parsimonious and theoretically clear model indicated there were two dimensions. We called the first dimension sensitization and the second dimension differentiation. Individuals appeared to be more or less sensitized to the idea that sexual media was pornographic, with some experiencing almost any type of sexual media as pornographic while others being less sensitized. Likewise, individuals were more or less likely to differentiate between different degrees of explicitness in sexual media.

Rather than adopting a traditional simple structure approach in an effort to obtain separate subsets or clusters of items (such as soft-core and hard-core pornography), we have used a twodimensional bi-factor model where all of the items reflect both dimensions. The first dimension is a unipolar general trait affecting all of the items, while the second is a bipolar trait contrasting responses to more sexually explicit media with responses to less sexually explicit media. We have proposed that people's judgments about pornography vary simultaneously along both dimensions, and that both processes affect their judgments. Other aspects of the descriptions in the items, such as the type of media (image, video, film, writing), the individuals depicted (man, woman, couple, etc.), or the acts involved (posing suggestively, intercourse, etc.), possibly influenced the responses, but the responses were governed largely by the two fundamental underlying dimensions of sensitization and differentiation.
Fig. 1 Final model using sample B. $N=1068$. All factor loadings are statistically significant, $p<.05$

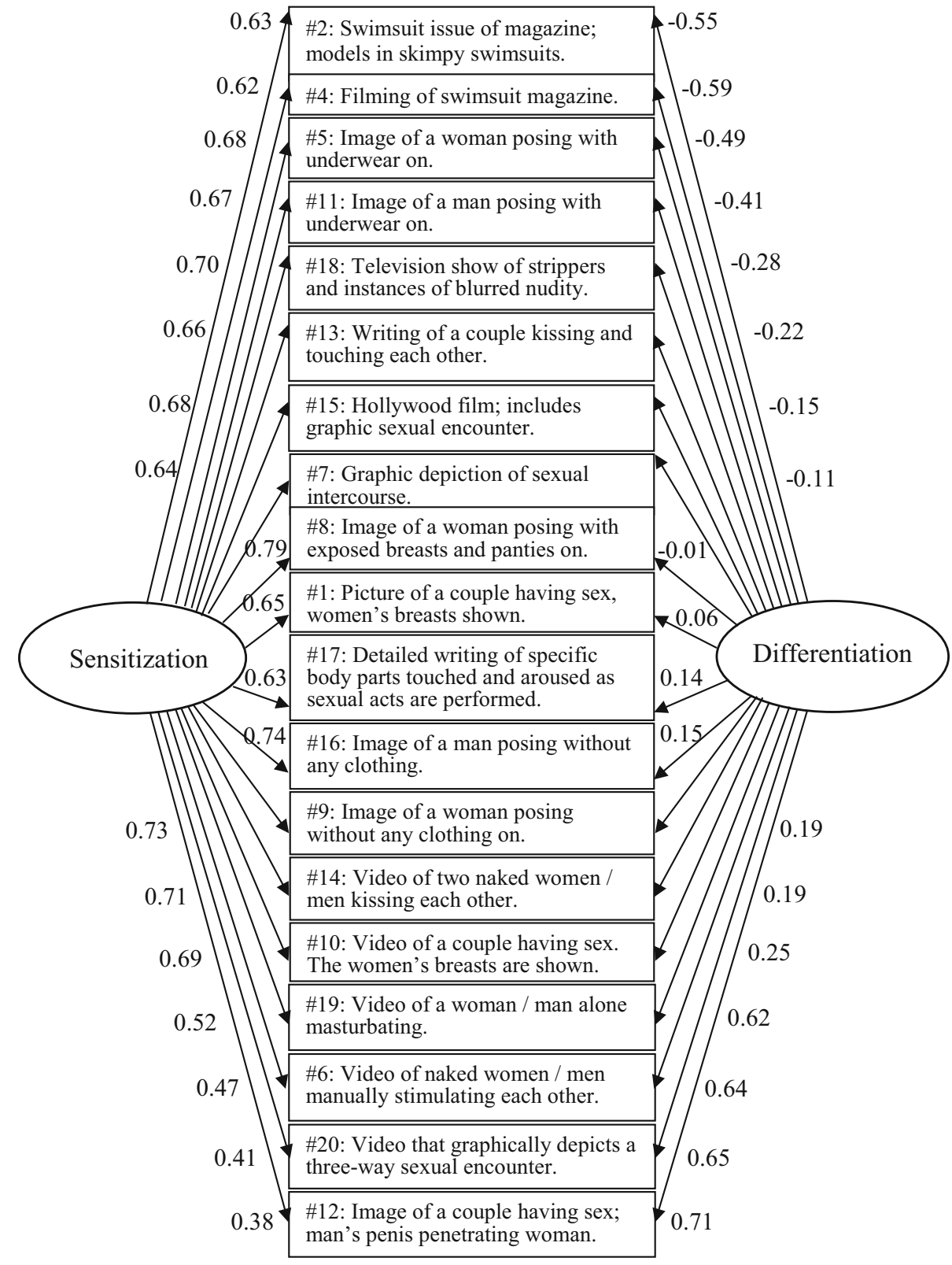


The identification of these dimensions is important, as now it is possible to carefully measure them with more advanced statistical software and procedures being available. Exploring the sensitization and differentiation dimensions can help us better understand the effects of pornography on individuals and relationships. It may be that one of these dimensions is better at predicting why some people and relationships seem to be influenced more negatively by pornography than others. It may also be that within couple differences or gender differences with these two dimensions are helpful when exploring the different usage patterns and effects for males and females (Poulsen et al., 2013). Is the fact that males and females view pornography at such different rates at least partially due to different levels of sensitization to pornography in the first place? Additionally, some researchers have distinguished different types of sexual media by classifying some of it as pornography and other material as erotica (Fisher \& Barak, 1991). While these distinctions may be useful for researchers in terms of exploring the differential effects of types of sexual media, it is not clear whether the general population makes such distinctions, and if as we found in this study pornography is multidimensional, it may be crucial to see how these two dimensions influence judgments about all types of sexual media.

\section{Limitations and Future Directions}

While our study advances what we know about the dimensionality of the concept of pornography, there are some limitations that suggest the need for additional research. The sample, though large and international, may have unexpected biases. Although the study results clearly showed two dimensions, we are aware that for sensitization (the factor that demonstrated the participants' tendency to rate all items higher or lower on the scale), the results could also be explained by participants' general response patterns. That is, certain participants might respond to surveys in a specific way, regardless of how sensitized they were to pornography. Also, since pornography is a fairly sensitive subject, we may expect response bias to exist or even influence the study outcome.

Although there were limitations to these data and findings, since we developed the model using the cross-validation procedure with a randomly selected half of our sample and verified it with the other half, we felt confident that the results indicated that the problem of over-fitting did not appear to exist. In other words, we believe that the two dimensions we discovered were not likely just based on potential participant response patterns or biases. Thus, we believe the results from this study may be applicable to other samples. Furthermore, it has been indicated in some studies that response bias could be reduced or lessened by adopting certain procedures such as administering computerized surveys instead of traditional surveys, providing a more anonymous environment, and using survey language that would be less likely to heighten the participants' sensitive reaction (e.g., Krumpal, 2013; Richman, Kiesler, Weisband, \& Drasgow, 1999). Although to our knowledge there is no known method to completely remove or prevent participant response bias, we utilized several of these suggested methods to hopefully reduce response pattern or bias.

For future research directions, we do hope that additional research showing the usefulness of these two dimensions will speak to the validity of the concepts with multiple samples. A nationally representative sample might improve the generalizability of these results. Additional research that gathers information on usage rates in addition to the perceptions we evaluated would also be an important next step. It may be that while certain types of sexual media are clearly considered pornographic, individuals may not view it often enough to be useful for the purposes of research evaluating the effects on individuals and relationships.

\section{Compliance with Ethical Standards}

Conflict of interest There were no conflicts of interest for any of the authors of this manuscript.

Ethical Approval All procedures performed in studies involving human participants were in accordance with the ethical standards of the institutional and/or national research committee and with the 1964 Declaration of Helsinki and its later amendments or comparable ethical standards.

Informed Consent Informed consent was obtained from all individual participants included in the study.

\section{Appendix: Perceptions Regarding Definition of Pornography}

Consider the statements below and indicate if you believe they describe what you would consider pornography.

1. A picture of a couple having sex, the women's breasts are shown but neither partner's genitalia are shown.

2. A swimsuit issue of a magazine showing models in skimpy swimsuits or strategically covered topless views in a variety of provocative poses.

3. A video showing a woman having sexual intercourse with an animal.

4. A television program showing the filming of a swimsuit edition of a magazine that shows models being filmed in a variety of provocative poses but no full nudity of breasts or genitalia.

5. An image of a woman alone posing in a suggestive way with underwear on.

6. A video showing two naked women or men manually stimulating each other.

7. A novel that includes one graphic depiction of sexual intercourse.

8. An image of a woman alone posing in a suggestive way with exposed breasts and panties on. 
9. An image of a woman alone posing in a suggestive way without any clothing on.

10. A short video depicting a couple having consensual sex. The women's breasts are shown but neither partner's genitalia are shown.

11. An image of a man alone posing in a suggestive way with only underwear on.

12. An image of a heterosexual couple having sex which shows the man's penis penetrating the woman.

13. A detailed description in writing of a couple passionately kissing and touching each other's clothed bodies and describing their arousal.

14. A video showing two naked women or men kissing each other.

15. A major Hollywood film or movie that includes one graphic sexual encounter.

16. An image of a man alone posing in a suggestive way without any clothing on.

17. A detailed description in writing of a couple engaging in foreplay and sex that includes mention of specific sexual body parts that are touched and aroused as sexual acts are performed.

18. A television show focused on strippers that includes multiple instances of blurred nudity.

19. A video of a woman or man alone masturbating.

20. A video that graphically depicts a three-way sexual encounter.

\section{References}

Amoroso, D. M., Brown, M., Pruesse, M., Ware, E. E., \& Pilkey, D. W. (1972). The effects of physiological measurement and presence of others on ratings of erotic stimuli. Canadian Journal of Behavioural Science, 4, 191-203.

Bollen, K. A. (1989). Structural equations with latent variables. New York, NY: Wiley.

Brown, T. A. (2015). Confirmatory factor analysis for applied research. New York, NY: The Guilford Press.

Brown, C., Anderson, J., Burggraf, L., \& Thompson, N. (1978). Community standards, conservatism, and judgments of pornography. Journal of Sex Research, 14, 81-95.

Chen, F. F., West, S. G., \& Sousa, K. H. (2006). A comparison of bifactor and second-order models of quality of life. Multivariate Behavioral Research, 41, 189-225.

D’Abreu, L. C. F., \& Krahé, B. (2014). Predicting sexual aggression in male college students in Brazil. Psychology of Men \& Masculinity, 15, 152162.

Dalecki, M. G., \& Price, J. (1994). Dimensions of pornography. Sociological Spectrum, 14, 205-219.

Dimitrov, D. M.(2012). Statistical methods for validation of assessment scale data in counseling and related fields. Alexandria, VA: American Counseling Association.
Fisher, W. A., \& Barak, A. (1991). Pornography, erotica, and behavior: More questions than answers. International Journal of Law and Psychiatry, $14,65-83$.

Hald, G. M., \& Mulya, T. W. (2013). Pornography consumption and nonmarital sexual behavior in a sample of young Indonesian university students. Culture, Health \& Sexuality, 15, 981-996.

Hald, G. M., Seaman, C., \& Linz, D. (2014). Sexuality and pornography. In D. L. Tolman \& L. M. Diamond (Eds.), APA handbook of sexuality and psychology (Vol. 2, pp. 3-35). Washington, DC: American Psychological Association.

Hartig, J., \& Höhler, J. (2008). Representation of competencies in multidimensional IRT models with within-item and between-item multidimensionality. Journal of Psychology, 216, 89-101.

Krumpal, I. (2013). Determinants of social desirability bias in sensitive surveys: A literature review. Quality \& Quantity, 47, 2025-2047.

Malamuth, N. M., Addison, T., \& Koss, M. (2000). Pornography and sexual aggression: Are there reliable effects and can we understand them? Annual Review of Sex Research, 11, 26-91.

Manning, J. C. (2006). The impact of internet pornography on marriage and the family: A review of the research. Sexual Addiction \& Compulsivity, 13, 131-165.

Poulsen, F. O., Busby, D. M., \& Galovan, A. M. (2013). Pornography use: Who uses it and how it is associated with couple outcomes. Journal of Sex Research, 50, 72-83.

Price, J., \& Dalecki, M. G. (1998). The social basis of homophobia: An empirical illustration. Sociological Spectrum, 18, 143-159.

Richman, W. L., Kiesler, S., Weisband, S., \& Drasgow, F. (1999). A metaanalytic study of social desirability distortion in computer-administered questionnaires, traditional questionnaires, and interviews. Journal of Applied Psychology, 84, 754-775.

Short, M. B., Black, L., Smith, A. H., Wetterneck, C. T., \& Wells, D. E. (2012). A review of Internet pornography use research: Methodology and content from the past 10 years. Cyberpsychology, Behavior, and Social Networking, 15, 13-23.

Sinković, M., Štulhofer, A., \& Božić, J. (2013). Revisiting the association between pornography use and risky sexual behaviors: The role of early exposure to pornography and sexual sensation seeking. Journal of Sex Research, 50, 633-641.

Subero, G. (2010). Gay Mexican pornography at the intersection of ethnic and national identity in Jorge Diestra's La Putiza. Sexuality and Culture, 14, 217-233.

Tsaliki,L. (2011). Playing with porn: Greek children's explorations in pornography. Sex Education, 11, 293-302.

Turnbull, D., \& Brown, M. (1977). Attitudes towards homosexuality and male and female reactions to homosexual and heterosexual slides. Canadian Journal of Behavioural Science, 9, 68-80.

Tylka, T. L. (2015). No harm in looking, right? Men's pornography consumption, body image, and well-being. Psychology of Men \& Masculinity, 16, 97-107.

Willoughby, B. J., \& Busby, D. M. (2016). In the eye of the beholder: Exploring variations in the perceptions of pornography. Journal of Sex Research, 53, 678-688.

Wright, P. J. (2013).U.S. males and pornography, 1973-2010: Consumption, predictors, correlates. Journal of Sex Research, 50, 60-71.

Wright, P. J., Tokunaga, R. S., \& Bae, S. (2014). More than a dalliance? Pornography consumption and extramarital sex attitudes among married U.S. adults. Psychology of Popular Media Culture, 3, 97-109.

Yu, C. K. (2013). Lust, pornography, and erotic dreams. Dreaming, 3, 175193. 